\title{
Análise da Viabilidade Técnica e Econômica da Implantação de um Sistema Solar Fotovoltaico em um Hotel na Cidade de Surubim
}

\author{
Analysis of the Technical and Economic Viability of the Implementation of a Solar \\ Photovoltaic System in a Hotel in the City of Surubim
}

Lucas França' ${ }^{1}$ https://orcid.org/0000-0001-8544-7810

Eduardo Sodré ${ }^{1}$ https://orcid.org/0000-0002-5793-8900

${ }^{1}$ Escola Politécnica de Pernambuco, Universidade de Pernambuco, Recife, Brasil

E-mail do autor principal: Lucas França lucasfranca@atonenergiasolar.com

\section{RESUMO}

A partir da edição da Resolução Normativa ANEEL N. 482/2012 a sociedade brasileira pode finalmente ter 0 direito de instalar painéis fotovoltaicos em suas fábricas, comércios e residências para gerar sua própria energia elétrica. No caso específico de hotéis e/ou motéis, a implantação de instalações para utilizar o sol como fonte de geração elétrica e para aquecimento de água vem a ter grande importância econômica para o estabelecimento. A conta de energia representa um alto percentual dos custos fixos desses estabelecimentos, que em geral possuem água aquecida em todos os banheiros, condicionadores de ar, frigobar e lâmpadas em todos os quartos. Este trabalho tem como objetivo fazer uma análise expedita da viabilidade técnico econômica da implantação de um sistema solar fotovoltaico em um hotel na cidade de Surubim. Como resultado da implantação deste projeto, o hotel conseguiu uma taxa interna de retorno de $26 \%$ em um intervalo de 25 anos, com fluxo de caixa positive a partir do $4^{\circ}$ ano pós-implantação do Sistema fotovoltaico.

PALAVRAS-CHAVE: Energia Solar; Fotovoltaica; Viabilidade Econômica; Hotéis;

\section{ABSTRACT}

Since the issuance of ANEEL Normative Resolution No. 482/2012 the Brazilian society may finally have the right to install photovoltaic panels in its factories, businesses and residences to generate its own electric energy. In the specific case of hotels and / or motels, the installation of facilities to use the sun as a source of electricity generation and for heating water is of great economic importance for the establishment. The energy bill represents a high percentage of the fixed costs of these establishments, which usually have heated water in all bathrooms, air conditioners, mini-bars and light bulbs in all rooms. This work aims to make an expedited analysis of the technical feasibility of the implantation of a solar photovoltaic system in a hotel in the city of Surubim. As a result of the implementation of this project, the hotel achieved an internal rate of return of $26 \%$ in an interval of 25 years, with positive cash flow from the 4th year post-implantation of the Photovoltaic System.

KEY-WORDS: Solar Energy; Photovoltaic; Economic Assesment; Hotels; 


\section{INTRODUÇÃO}

O setor de energia elétrica é base fundamental para o desenvolvimento mundial, sendo a natureza da geração dessa energia alvo de muitas discussões, principalmente nos últimos anos, conforme a população mundial vem crescendo e a preocupação com a sustentabilidade e com os impactos ambientais que a humanidade gera ao produzir energia [1].

Dessa forma, a busca por novas fontes de energia, com foco maior nas fontes renováveis, é extremamente necessária para fazer frente ao crescimento populacional mundial e ao consequente aumento do consumo de energia, tanto para a indústria, como para fins residenciais.

Paralelo a esse cenário, as fontes de combustíveis fósseis se esgotam rapidamente em todo o mundo, e acordos diversos firmados em conferências mundiais demonstram a vontade política e, acima de tudo, o compromisso mundial por mudar a matriz energética para fontes consideradas limpas e renováveis.

No Brasil, possuímos atualmente uma das matrizes elétricas mais limpas do mundo, principalmente pelo aproveitamento do potencial hidráulico dos nossos rios. Contudo, o potencial hidráulico já está praticamente todo utilizado, além de nos últimos anos, estarmos constantemente sofrendo com cenários de seca. E Esses cenários constantes de depleção dos níveis das usinas hidráulicas têm levando o ONS a utilizar muitas usinas térmicas, com consequentemente acréscimos nas tarifas de energia pelo maior custo de operação destas.

Dessa forma, as energias ditas alternativas, como a eólica e a solar, surgem como excelentes caminhos para diversificação da matriz e para o atendimento ao consumo crescente, principalmente aqui no Brasil, onde temos grandes extensões de terra extremamente favoráveis em termos de vento e irradiação solar.

As usinas eólicas já se mostraram viáveis técnica e economicamente, principalmente na Região Nordeste do país, com o modelo de geração concentrada, e a energia sendo vendida no Ambiente de Contratação Regulado - ACR, possuindo preço altamente competitivo com as outras fontes.

A energia proveniente do sol pode ser aproveitada de diversas maneiras, dentre as principais aplicações estão o aquecimento da água para consumo, o aquecimento de água ou outros fluidos para processos nas indústrias e o aproveitamento da irradiação para geração direta de corrente elétrica através do efeito fotovoltaico.

Dentre os principais benefícios do uso da fonte solar, estão a natureza inesgotável da fonte (sol), o custo zero com uso de combustíveis, a previsibilidade em base anual da quantidade de sol, e no caso do Nordeste, a alta incidência no território da região.

A energia fotovoltaica, assim como os projetos para aquecimento de água para 0 consumo, tem como vocação uma característica interessante, que é a desconcentração da geração. Essas modalidades podem, e têm sido, instaladas diretamente nos locais de consumo, sejam indústrias, estabelecimentos comerciais ou residenciais.

No caso específico de hotéis e/ou motéis, a implantação de instalações para utilizar o sol como fonte de geração elétrica e para aquecimento de água vem a ter grande importância econômica para o estabelecimento. A conta de energia representa um alto percentual dos custos fixos desses estabelecimentos, que em geral possuem água aquecida em todos os banheiros, condicionadores de ar, frigobar e lâmpadas em todos os quartos [2, 3].

\section{PROJETO DO SISTEMA FOTOVOLTAICO}

Foram colhidas informações reais do empreendimento alvo da análise, tais como: os projetos de arquitetura; as doze últimas contas de energia; taxa mínima de atratividade, etc. Usamos o software PVSOL para projetar o sistema fotovoltaico de acordo com o consumo médio. Levamos orçamentos reais para a compra dos equipamentos, e mão-de-obra de instalação, projeto, etc.

O Hotel possui 42 quartos-dormitórios, distribuídos em quatro blocos de edificações com cobertas em telha cerâmicas 02 águas, mais um bloco maior, que contempla espaços de recepção, auditório e restaurante. Este bloco também possui 02 águas de coberta, e foi o escolhido para a instalação dos módulos fotovoltaicos, pelos motivos que serão descritos mais à frente.

A modalidade tarifária de cobrança atual da energia consumida pelo hotel é denominada A4 Horo-sazonal verde, sendo cliente CELPE. Para esta modalidade, são aplicadas tarifas diferentes 
para os horários de ponta e fora ponta, mas utiliza valor único na cobrança da demanda contratada, que nesse caso é de 40kVA [4].

De acordo com a Resolução Normativa no $414 / 2010$, o consumidor pode escolher ser faturado na modalidade " $B$ " desde que a potencia nominal total dos transformadores seja igual ou inferior a 112,5 kVA. Essa opção foi feita pelo hotel, visando eliminar a obrigação de pagar a demanda contratada, tendo em vista a futura instalação da usina de geração fotovoltaica [5].

Criamos uma planilha no Excel para simular o fluxo de caixa do investimento até 25 anos de funcionamento do sistema, incluindo taxas de juros real do financiamento escolhido, reinvestimento para manutenção e/ou troca de equipamentos com base na vida útil dos mesmos. Calculamos a TIR dos projetos e vamos comparar se esta supera TMA do empreendimento.

Por haver disponibilidade de área de coberta no Hotel para a instalação dos módulos fotovoltaicos, optou-se por essa opção por possuir também um custo menor que a opção de instalar sobre o solo. A Figura 1. demonstra o consumo do cliente aferido com base nas contas de energia disponibilizadas.

Com base na Figura 1, o consumo anual de energia do cliente foi de $112.000 \mathrm{kWh} / \mathrm{ano}$. Na Figura 2 temos os dados gerais de irradiação utilizados para o cálculo da quantidade de módulos fotovoltaicos necessários para gerar essa quantidade de energia necessária para o hotel. A fonte utilizada pelo software PVSOL é o MeteoSyn.

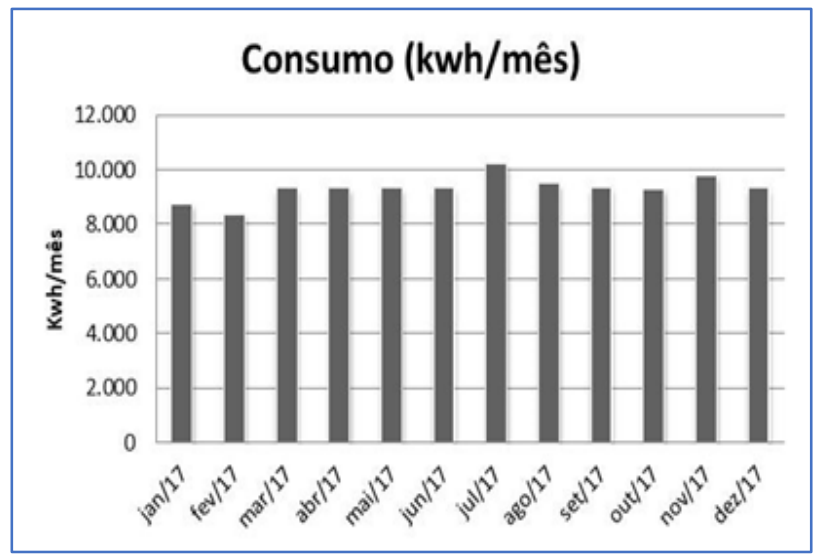

Figura 1: Consumo de energia do Hotel em 2017. Fonte: Os Autores.

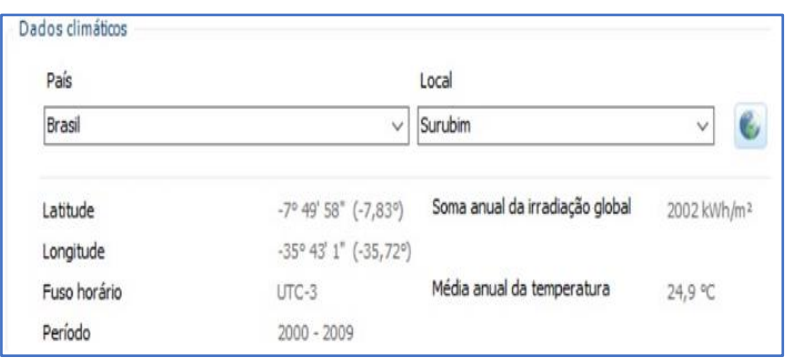

Figura 2: Dados de irradiação de Surubim. Fonte: (software PVSOL).

Considerando uma perspectiva de futura expansão, o hotel solicitou ao projetista que fosse realizado um projeto no qual a energia gerada fosse $5 \%$ maior que a energia utilizada atualmente. Dessa forma, utilizamos o valor de $118.000 \mathrm{kWh} /$ ano de geração. Com base nos dados de irradiação anual direta, difusa, albedo; inclinação e orientação dos módulos na coberta; escolha do módulo; sombreamento; perdas; etc; - cálculo do PVSOL determina que serão necessários 236 módulos fotovoltaicos do modelo escolhido, tal qual segue na Figura 3.

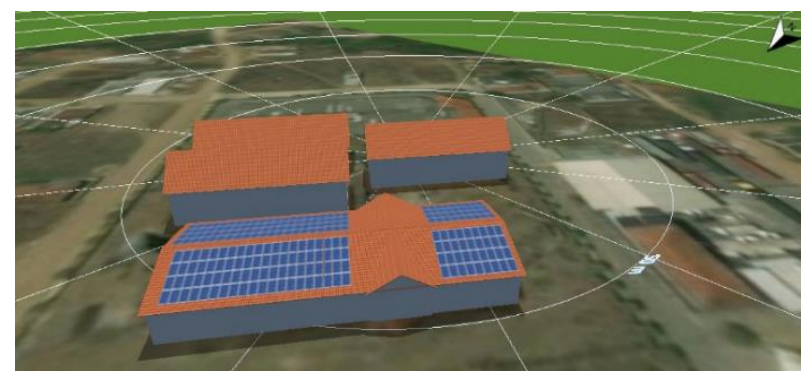

Figura 3: Cobertas do Hotel moduladas em 3D no PVSOL com disposição dos 236 módulos FV. Fonte: (software PVSOL).

O arranjo foi distribuído da seguinte forma, de acordo com os limites de tensão e corrente máximas de entrada e de operação dos inversores:

Tabela 1: Resumo de equipamentos do Sistema Fotovoltaico que será implantado

\begin{tabular}{|c|c|c|c|}
\hline \multicolumn{4}{|c|}{ TABELA RESUMO SISTEMA FV HOTEL } \\
\hline INVERSORES & $\begin{array}{c}\text { NoSTRINGSX } \\
\text { NoSTRINGSX }\end{array}$ & FATOR DE \\
(MODELO/POTENCIA) & MÓDULOS & MÓDULOS & DIMENSION \\
MPPT1 & MPPT2 & AMENTO \\
1X PHB 25 KW & $4 \times 21$ & N/A & $109 \%$ \\
1XPHB 25 KW & $4 \times 21$ & N/A & $109 \%$ \\
1X PHB 20 KW & $2 \times 17$ & $2 \times 17$ & $111 \%$ \\
\hline \multicolumn{2}{|c|}{ POTENCIA TOTAL INVERSORES (KW) } & 70,0 \\
POTENCIA TOTAL MÓDULOS (KW) & 76,7 \\
\hline
\end{tabular}

Fonte: Os Autores. 
O fator de dimensionamento do inversor, mostrado na Tabela 1., é um índice importante para verificar a eficiência do projeto. Este dimensionamento se dá pela divisão da potência total dos módulos fotovoltaicos do projeto, divido pela potência nominal do inversor. Em regra, é positivo que este valor esteja acima de 100\%, pois devem ser consideradas perdas de potência dos painéis na instalação, além de otimizar o uso dos inversores ao longo das horas de sol, tornando ainda o custo do projeto mais barato.

A lista resumida dos equipamentos que serão utilizados para a realização da instalação está descritas conforme Tabela 2.

Tabela 2: Lista de equipamentos

\begin{tabular}{|c|c|c|}
\hline Item & Qte. & Descrição \\
\hline 1 & 2 & Inversor Fotovoltaico PHB 25KW com wi fi \\
\hline 2 & 1 & Inversor Fotovoltaico PHB 20KW com wi fi \\
\hline 3 & 236 & Módulo FV Jinko Solar Policristalino 325Wp \\
\hline 4 & - & $\begin{array}{l}\text { Estrutura de suporte em alumínio mais acessórios } \\
\text { de fixação }\end{array}$ \\
\hline 5 & 3 & StringBox CC 4 entradas/4 saídas \\
\hline 6 & 1 & Quadro de Conexão de inversores e Proteção CA \\
\hline 7 & 230 & Cabo Solar 1000Vcc Prot.UV Preto 4,0 $\mathrm{mm}^{2}$ \\
\hline 8 & 230 & Cabo Solar 1000Vcc Vermelho Prot. UV 4,0 $\mathrm{mm}^{2}$ \\
\hline
\end{tabular}

Fonte: Os autores.

\section{Análise da Viabilidade Técnico- econômica}

A decisão de realizar um investimento em um projeto e posterior execução de um sistema fotovoltaico em qualquer empreendimento deve ser precedida sempre de uma profunda análise técnico-econômica, baseada nos custos, tempo de retorno, etc. O resultado dessa análise dará ao investidor as ferramentas necessárias para tomar a decisão devida. No caso específico do escopo deste artigo, a motivação vem da possibilidade real de o empresário conseguir diminuir seu custo fixo, através de uma redução de mais de $90 \%$ do valor pago mensalmente à concessionária de energia.

O estudo de um projeto de geração fotovoltaica é composto da análise do potencial e da viabilidade econômico-financeira. A parte técnica do estudo de viabilidade está associada à capacidade de geração e da irradiação média anual de determinada região. Através do estudo de viabilidade econômico-financeira é possível determinar se um projeto é interessante para o investidor.

Nesta pesquisa, será considerado um fluxo de caixa discretizado anualmente, os investimentos, que corresponde ao capital próprio e as entradas de caixa ocorrem em períodos subseqüentes.

Contudo, o VPL (Valor Presente Líquido) será calculado considerando o Fluxo de Caixa Descontado. Portanto, para efeito de estudo, o Custo de Capital Próprio será fixado em $10 \%$.

A decisão de implantação de um projeto deve considerar:

- critérios econômicos: rentabilidade do investimento;

- critérios financeiros: disponibilidade de recurso;

- critérios imponderáveis: fatores não conversíveis em dinheiro.

A análise econômica para ser consistente deve considerar princípios básicos que irão nortear o trabalho, como segue: é imprescindível que exista pelo menos duas alternativas para o investidor; o objeto de estudo deve ser convertido em dinheiro; os desvios devem ser significativos; o custo de oportunidade sempre será considerado; o valor de mercado é o que realmente importa no estudo econômico [6].

A análise econômica, financeira, da instalação fotovoltaico será elaborada utilizando uma previsão do fluxo de caixa e definição da taxa de desconto. Posteriormente, os cálculos que definirão os índices determinísticos serão: VPL (Valor Presente Líquido) e TIR (Taxa Interna de Retorno).

O fluxo de caixa é um instrumento utilizado pelos especialistas da área econômico-financeira, objetivando avaliar se determinado projeto tem capacidade de remunerar o capital aplicado. Esta análise pode ser direcionada para avaliar o empreendimento, neste caso interessa aos órgãos financeiros para medir a capacidade de pagamento do empréstimo ou focar na taxa de retorno do recurso próprio alocado ao projeto, esta é que determina se o projeto tem capacidade de remunerar o investidor [6].

Um projeto, no entanto, pode ser no mínimo, decomposto em duas partes, o fluxo do financiamento de longo prazo e o fluxo do empresário, admitindo-se parte do projeto sendo financiado por bancos e a outra parte pelos recursos próprios.

O objetivo do estudo de caso passa e ser então, de forma prática e direta:

1. descobrir se há viabilidade técnicoeconômica de um Hotel investir em um sistema de energia fotovoltaico com base em uma taxa mínima de atratividade estabelecida pelo investidor;

2. estabelecer a viabilidade técnicoeconômica da implantação de um sistema 
fotovoltaico já considerando que com a implantação do sistema FV o consumidor (Hotel) irá voltar para a modalidade B3 comercial trifásico (CELPE);

3. Projetar sistema fotovoltaico com base no consumo dos últimos doze meses do Hotel:

4. Simular fluxo de caixa do investimento e retorno para implantação do projeto, com recursos oriundos de financiamento do FNESOL do Banco do Nordeste;

5. Calcular a taxa interna de retorno - TIR do projeto;

6. Comparar com a TMA do investidor.

\section{RESULTADOS}

Com base na irradiação calculada pelo software PVSOL para o local (Surubim-PE), temos que a economia mensal em Reais, por mês, se dá da seguinte forma no primeiro ano: $112.000 \mathrm{kWh} / \mathrm{ano}$ (produção esperada do sistema FV instalado) $\times \mathrm{R} \$ 0,7392771$ (valor da tarifa CELPE para o grupo "B") $=R \$ 82.799,03$.

Essa economia divididaem 12 meses significa uma economia média mensal de $\mathrm{R} \$ 6.899,92$. Esse valor inclusive servirá como limite superior para se estabelecer o valor da parcela inicial do financiamento.

Foi estabelecido um roteiro, com base nos dados colhidos via projeto e orçamento, a fim de se estabelecer os índices desejados neste estudo e posterior verificação da viabilidade técnicoeconômica da implantação do projeto:

Tabela 3: Roteiro de Viabilidade financeira.

\begin{tabular}{|c|c|c|}
\hline Investimento & $314.781,79$ & R\$ \\
\hline IPCA (IBGE) & 0,0374 & mês \\
\hline Taxa de juros BNB & 0,0168 & a.a. \\
\hline $\begin{array}{c}\text { Taxa de juros final } \\
\text { Taxa de juros final }\end{array}$ & 0,054 & a.a. \\
\hline $\begin{array}{c}\text { Taxa de juros final com } \\
\text { bônus de adimplência }\end{array}$ & 0,0045 & a.m. \\
\hline $\begin{array}{c}\text { Taxa de juros final com } \\
\text { bônus de adimplência }\end{array}$ & 0,004 & a.a. \\
\hline $\begin{array}{c}\text { Prazo do financiamento } \\
\text { Tipo de amortização }\end{array}$ & 66 & mês \\
\hline $\begin{array}{c}\text { Previsão de economia } \\
\text { média mensal }\end{array}$ & $6.899,92$ & R\$ \\
\hline $\begin{array}{c}\text { Taxa mínima de } \\
\text { atratividade }\end{array}$ & $10 \%$ & a.a. \\
\hline $\begin{array}{c}\text { Valor médio da conta de } \\
\text { energia }\end{array}$ & $7.084,97$ & R\$ \\
\hline
\end{tabular}

Fonte: Os Autores.

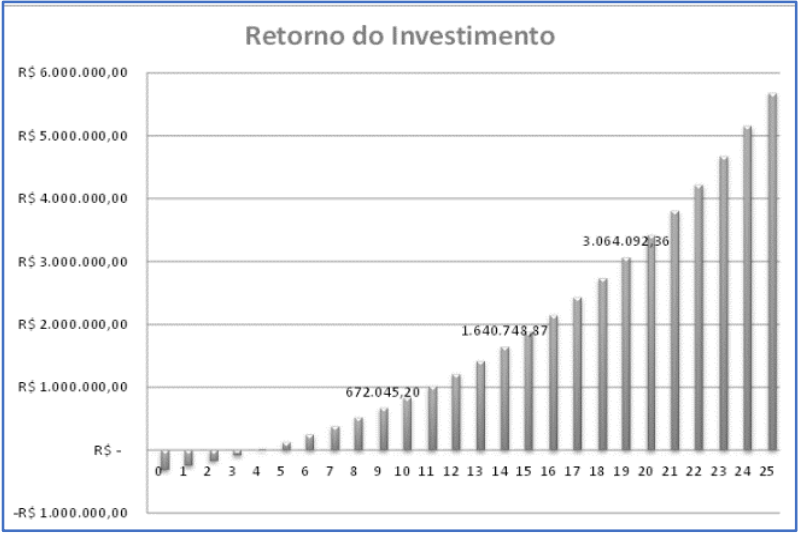

Figura 4: Payback simples. Fonte: Os Autores.

Realizando uma simulação de retorno do investimento, considerando apenas o valor do projeto disponibilizado em sua totalidade pela instituição financeira e o valor economizado com as contas de energia, temos a Figura 4. Onde são considerados valores da conta de energia do cliente no grupo B3, apenas as parcela referente à energia, sem iluminação pública e com inflação anual de $8 \%$, retirados os juros pagos em cada ano no período do financiamento.

Da Figura 4. aferimos que ao fim do ano 4 após o início do pagamento das parcelas, o cliente já terá coberto o custo do investimento realizado pela instituição financeira e já há economia de $R \$$ $16.202,88$.

Se considerarmos da mesma forma o valor financiado, a economia com a redução das contas de energia, mas inserindo os juros pagos no período, além de uma substituição de todos os inversores a cada 10 anos de operação, custos com O\&M (operação e manutenção), temos a Figura 5. como sendo o payback descontado ao longo da vida útil considerada do projeto:

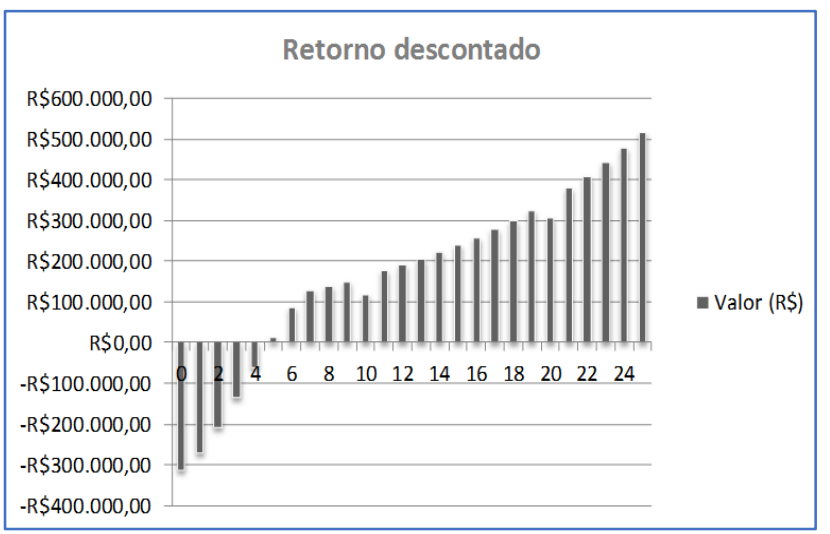

Figura 5: Payback descontado. Fonte: Os Autores. 
A seguir, no resumo do fluxo de caixa elaborado para este projeto, teremos mais parâmetros acerca da viabilidade do investimento no universo de 25 anos de vida útil, conforme Tabela 4.

O investidor (cliente), forneceu uma taxa mínima de atratividade (TMA) de 10\%, o que significa que para tomar a decisão de investir, o projeto sugerido obrigatoriamente teria que oferecer um retorno anual superior a esse valor. Para o caso estudado, esse valor foi definido justamente pela taxa interna de retorno (TIR), onde foi obtida nos 25 anos de duração, uma taxa de $24 \%$.
Através da análise de viabilidade econômica do projeto, pode-se afirmar que ele é viável e apresenta um bom custo-benefício, já que ele possui um VPL positivo e um ROI (Retorno Sobre o investimento) que mostra que durante a vida útil do projeto o sistema trará um retorno 3,92 vezes maior que o valor investido. $O$ investimento também mostra uma TIR de $24 \%$, mais que 0 triplo da rentabilidade anual de uma poupança. A seguir, a fórmula de cálculo do ROI, na equação 1:

$$
R O I=\left(\frac{\text { Receita-Custo }}{\text { Custo }}\right) \times 100
$$

Tabela 4: Fluxo de Caixa do Investimento.

\begin{tabular}{|c|c|c|c|c|c|c|}
\hline Ano & $\begin{array}{c}\text { Valor } \\
\text { financimento }\end{array}$ & $\begin{array}{l}\text { Juros com bônus } \\
\text { de adimplência }\end{array}$ & $\begin{array}{c}\text { Gastos com } \\
\text { Conta de Energia }\end{array}$ & $0 \& \mathrm{M}$ & Receita & Fluxo de Caixa \\
\hline $\mathbf{O}$ & $\mathrm{R} \$ 314.781,79$ & & & & -R\$314.781,79 & -R\$314.781,79 \\
\hline 1 & $\mathrm{R} \$ 283.303,61$ & $\mathrm{R} \$ 14.786,92$ & $R \$ 22.064,91$ & $\mathrm{R} \$ 700,00$ & $R \$ 48.299,44$ & $-R \$ 335.512,36$ \\
\hline 2 & $\mathrm{R} \$ 220.347,25$ & R\$11.711,85 & $\mathrm{R} \$ 1.166,40$ & $\mathrm{R} \$ 756,00$ & $\mathrm{R} \$ 89.422,96$ & $\mathrm{R} \$ 12.832,36$ \\
\hline 3 & R\$157.390,90 & $\mathrm{R} \$ 8.814,07$ & $\mathrm{R} \$ 1.259,71$ & $\mathrm{R} \$ 816,48$ & $R \$ 96.576,80$ & $R \$ 22.730,18$ \\
\hline 4 & $\mathrm{R} \$ 94.434,54$ & $\mathrm{R} \$ 5.916,30$ & $\mathrm{R} \$ 1.360,49$ & $R \$ 881,80$ & $\mathrm{R} \$ 104.302,94$ & $\mathrm{R} \$ 33.188,00$ \\
\hline 5 & $\mathrm{R} \$ 31.478,18$ & $\mathrm{R} \$ 3.018,52$ & $\mathrm{R} \$ 1.469,33$ & $R \$ 952,34$ & $\mathrm{R} \$ 112.647,18$ & $R \$ 44.250,63$ \\
\hline 6 & $\mathrm{R} \$ \mathrm{O}, \mathrm{OO}$ & $R \$ 422,59$ & $\mathrm{R} \$ 1.586,87$ & $\mathrm{R} \$ 1.028,53$ & $R \$ 121.658,95$ & $\mathrm{R} \$ 87.142,78$ \\
\hline 7 & R\$O,OO & & $\mathrm{R} \$ 1.713,82$ & $\mathrm{R} \$ 1.110,81$ & $\mathrm{R} \$ 131.391,67$ & $\mathrm{R} \$ 128.567,03$ \\
\hline 8 & $\mathrm{R} \$ \mathrm{O}, \mathrm{OO}$ & & $\mathrm{R} \$ 1.850,93$ & R\$1.199,68 & $\mathrm{R} \$ 141.903,00$ & $\mathrm{R} \$ 138.852,40$ \\
\hline 9 & R\$O,OO & & R\$1.999,00 & R\$1.295, 65 & R\$153.255, 24 & $R \$ 149.960,59$ \\
\hline 10 & $\mathrm{R} \$ \mathrm{O}, \mathrm{OO}$ & & $R \$ 2.158,92$ & $\mathrm{R} \$ 47.100,00$ & $\mathrm{R} \$ 165.515,66$ & $\mathrm{R} \$ 116.256,74$ \\
\hline 11 & $\mathrm{R} \$ \mathrm{O}, \mathrm{OO}$ & & $\mathrm{R} \$ 2.331,64$ & R\$1.399, 30 & $R \$ 178.756,92$ & $\mathrm{R} \$ 175.025,97$ \\
\hline 12 & R\$O,OO & & $\mathrm{R} \$ 2.518,17$ & $\mathrm{R} \$ 1.511,25$ & $\mathrm{R} \$ 193.057,47$ & R\$189.028,05 \\
\hline 13 & R\$O,OO & & $\mathrm{R} \$ 2.719,62$ & $\mathrm{R} \$ 1.632,15$ & $\mathrm{R} \$ 208.502,07$ & $\mathrm{R} \$ 204.150,30$ \\
\hline 14 & R\$O,OO & & $\mathrm{R} \$ 2.937,19$ & $\mathrm{R} \$ 1.762,72$ & $R \$ 225.182,23$ & $\mathrm{R} \$ 220.482,32$ \\
\hline 15 & R\$O,OO & & $\mathrm{R} \$ 3.172,17$ & $\mathrm{R} \$ 1.903,74$ & $R \$ 243.196,81$ & $\mathrm{R} \$ 238.120,91$ \\
\hline 16 & R\$O,OO & & $\mathrm{R} \$ 3.425,94$ & $\mathrm{R} \$ 2.056,04$ & $R \$ 262.652,56$ & $R \$ 257.170,58$ \\
\hline 17 & $\mathrm{R} \$ \mathrm{O}, \mathrm{OO}$ & & $\mathrm{R} \$ 3.700,02$ & $\mathrm{R} \$ 2.220,52$ & $R \$ 283.664,76$ & $R \$ 277.744,23$ \\
\hline 18 & R\$O,OO & & R\$3.996,02 & $\mathrm{R} \$ 2.398,16$ & $R \$ 306.357,94$ & $R \$ 299.963,76$ \\
\hline 19 & $\mathrm{R} \$ \mathrm{O}, \mathrm{OO}$ & & $\mathrm{R} \$ 4.315,70$ & $\mathrm{R} \$ 2.590,01$ & $R \$ 330.866,58$ & $R \$ 323.960,86$ \\
\hline 20 & R\$O,OO & & $R \$ 4.660,96$ & $R \$ 47.100,00$ & $\mathrm{R} \$ 357.335,90$ & $\mathrm{R} \$ 305.574,95$ \\
\hline 21 & $\mathrm{R} \$ \mathrm{O}, \mathrm{OO}$ & & $R \$ 5.033,83$ & $\mathrm{R} \$ 2.797,21$ & $R \$ 385.922,78$ & $\mathrm{R} \$ 378.091,73$ \\
\hline 22 & R\$O,OO & & $\mathrm{R} \$ 5.436,54$ & R\$3.020,99 & $R \$ 416.796,60$ & $R \$ 408.339,07$ \\
\hline 23 & $\mathrm{R} \$ \mathrm{O}, \mathrm{OO}$ & & $\mathrm{R} \$ 5.871,46$ & $R \$ 3.020,99$ & $\mathrm{R} \$ 450.140,33$ & $\mathrm{R} \$ 441.247,87$ \\
\hline 24 & R\$O,OO & & R\$6.341, 18 & $R \$ 3.474,14$ & $R \$ 486.151,55$ & $R \$ 476.336,23$ \\
\hline 25 & $\mathrm{R} \$ \mathrm{O}, \mathrm{OO}$ & & $\mathrm{R} \$ 6.848,48$ & $R \$ 3.474,14$ & $R \$ 525.043,68$ & $\mathrm{R} \$ 514.721,06$ \\
\hline
\end{tabular}

Fonte: Os Autores.

Os índices financeiros calculados estão apresentados na Tabela 5.

Tabela 5: Índices Financeiros.

\begin{tabular}{|l|r|}
\hline T.M.A. & $10 \%$ \\
\hline VPL & R\$866.611,53 \\
\hline TIR & $24 \%$ \\
\hline
\end{tabular}

Fonte: Os Autores.

\section{CONCLUSÃO}

O sistema em questão pode atender de forma satisfatória a outros consumidores residenciais que pretendem implementar uma microgeração, já que o investimento se paga antes que os equipamentos atinjam sua vida útil. E o retorno aconteceu em um tempo relativamente curto (em 5 anos no payback descontado), para sistemas fotovoltaicos.

Assim sendo, este trabalho pode servir de apoio a consumidores que desejam instalar uma microgeração fotovoltaica e precisem saber 0 payback do investimento e a rentabilidade anual do sistema.

Com esses indicadores financeiros conclui-se que o objetivo do trabalho foi atingido.

Apesar de o Brasil possuir excelentes índices de irradiação, o que favorece a geração de energia solar, a cadeia produtiva da tecnologia 
fotovoltaica ainda se concentra no exterior, o que eleva os custos de sistemas fotovoltaicos.

\section{REFERÊNCIAS}

[1] CAPRA, F.; LUISI, P. L. The Systems View of Life: A Unifying Vision. New Yorke:

Cambridge University Press, 2014.

[2] G. FILHO, J. et al. Traditional Photovoltaic System and Hybrid Photovoltaic System: a comparative study for a residence. In: SIMPÓSIO BRASILEIRO DE SISTEMAS ELÉTRICOS, 7., 12-16 maio 2018, Niterói, RJ. Anais [...] Nietrói, RJ: UFF, 2018.

[3] OLIVEIRA, H. M. de; SODRÉ, E. de A. Study of a Grid-Tied Photovoltaic System in Caruaru using PVsyst and Skelion. In: SIMPÓSIO BRASILEIRO DE SISTEMAS ELÉTRICOS, 7., 12-16 maio 2018, Niterói, RJ. Anais [...] Nietrói, RJ: UFF, 2018.

[4] CELPE. Opções Tarifárias. Disponível em: http://servicos.celpe.com.br/residencialrural/Pages/Alta\%20Tens\%C3\%A3o/opcoestarifarias.aspx. Acesso em 23 ago. 2018 às $19 \mathrm{~h} 20$.

[5] ANEEL. Resolução Normativa 414/2010. Brasília: Agência Nacional de Energia Elétrica, 2010.

[6] CASAROTTO FILHO, N.; KOPITTKE, H. B. Análise de Investimentos. 10. ed. São Paulo: Atlas, 2010. 